\title{
BRST-BV quantization of gauge theories with global symmetries
}

\author{
I. L. Buchbinder ${ }^{1,2,3, a}$, P. M. Lavrov ${ }^{1,2, b}$ \\ ${ }^{1}$ Center of Theoretical Physics, Tomsk State Pedagogical University, Kievskaya St. 60, 634061 Tomsk, Russia \\ 2 National Research Tomsk State University, Lenin Av. 36, 634050 Tomsk, Russia \\ ${ }^{3}$ Departamento de Física, ICE, Universidade Federal de Juiz de Fora, Campus Universitário, Juiz de Fora, MG 36036-900, Brazil
}

Received: 28 February 2018 / Accepted: 17 June 2018 / Published online: 26 June 2018

(C) The Author(s) 2018

\begin{abstract}
We consider the general gauge theory with a closed irreducible gauge algebra possessing the nonanomalous global (super)symmetry in the case when the gauge fixing procedure violates the global invariance of classical action. The theory is quantized in the framework of BRST-BV approach in the form of functional integral over all fields of the configuration space. It is shown that the global symmetry transformations are deformed in the process of quantization and the full quantum action is invariant under such deformed global transformations in the configuration space. The deformed global transformations are calculated in an explicit form in the one-loop approximation.
\end{abstract}

\section{Introduction}

BRST quantization procedure, initiated in the works [1-8], which can be realized within the Hamiltonian BFV approach $[9,10]$ or within the Lagrangian BV approach $[11,12]$, is a powerful and universal tool to formulate the quantum gauge theories and investigate their structures. This procedure is applicable to an extremely wide class of gauge theories including the (super) Yang-Mills theories, (super)gravity, (super)strings and more specific gauge theories like the gauge antisymmetric field models. All these theories possess many common properties therefore one can talk about a general gauge theory and study its quantum aspects, including the renormalization issues (see e.g. [13-15]), in general terms.

Besides the local symmetries, many gauge theories are characterized by the rigid symmetries. For example, the role which is played by the global chiral symmetry in the Standard Model (see e.g. [16]) or the role which is played by global conformal group in the String Theory (see e.g. [17]) are well known. In many cases it is essential to preserve the classical global symmetry in quantum theory since in the opposite

\footnotetext{
a e-mail: joseph@tspu.edu.ru

be-mail: lavrov@tspu.edu.ru
}

case the important physical properties of the theory can be violated. Example of such a model is $\mathcal{N}=4$ supersymmetric Yang-Mills theory, where the classical rigid superconformal symmetry is conserved at quantum level (see e.g. [18]). As a result, we arrive at a general problem to study the classical global symmetries in BRST quantization of the gauge theory.

A specific aspect of the above general problem arises in supersymmetric gauge theories. These theories possess a gauge symmetry and rigid or global supersymmetries. Such theories can be formulated either in the component formalism or in the terms of superfields. In the first case the supersymmetry is non manifest and when we quantize the corresponding gauge theory imposing the gauge fixing conditions on vector fields we violate the rigid supersymmetry. Therefore it is unclear if the quantum effective action should be supersymmetric. At first, this problem arose in $\mathcal{N}=1$ super YangMills theories and led to the study of the aspects of global symmetry in quantum gauge theories [19-22] However, this problem was automatically resolved after formulation of the $\mathcal{N}=1$ supergauge theories in terms of $\mathcal{N}=1$ superfields (see e.g. [23]), where the manifestly $\mathcal{N}=1$ gauges have been used. However, the problem is still remains in extended super Yang-Mills theories. At present, the best formulation of $4 D, \mathcal{N}=4$ rigid super Yang-Mills theory is achieved in terms of $4 D, \mathcal{N}=2$ or in terms of $4 D, \mathcal{N}=3$ harmonic superfields [24]. In this case only part of supersymmetries are manifest, the other remain hidden. The same situation will be in $6 D, \mathcal{N}=(1,1)$ rigid super Yang-Mills theory, which is similar in many aspects to $4 D, \mathcal{N}=4$ super Yang-Mills theory. At present, the best formulation of such a theory is achieved in terms of $6 D, \mathcal{N}=(1,0)$ superfields (see e.g. [29] and references therein). Again, one part of supersymmetries is manifest and the other part is hidden. In all such theories the gauges preserve the manifest symmetries and violate the hidden supersymmetries (see e.g. [25-28,30,31]).

Another aspect of the same problem arises at quantization of $4 D, \mathcal{N}=2$ superconformal theories where the gauges 
preserve the manifest $\mathcal{N}=2$ supersymmetry but violate the global superconformal invariance. This aspect was studied in the series of the papers [32-34] where an approach to general problem of global symmetries in quantum gauge theories was proposed and it was shown that under some assumptions concerning the structure of the theory, the quantization leads to deformation of global symmetries and, in particular, to deformation of superconformal transformations.

In this paper we study the above problem in general terms and generalize all the previous results. We consider the general gauge theory possessing the non-anomalous global symmetry and quantize this theory in the framework of the BRST$\mathrm{BV}$ procedure. It is assumed that gauges violate the initial global symmetry of the classical action. Under these conditions we develop a maximally general approach to the structure of the global symmetries at the quantization of gauge theories. We show like in work [32] that the quantization procedure leads to deformation of classical global transformations. However, we use the more general effective action then in [32] that provides more possibilities for purely algebraic analysis of the global symmetries in quantum gauge theory. We prove that although the vacuum functional is invariant under classical global transformations, the effective action is not invariant and its invariance requires the quantum corrections to generators of global symmetry transformations. The maximally general form of such deformations is derived. In particular, we prove that the full quantum action in the functional integral is invariant under the deformed transformations. The one-loop invariance is studied in detail and the corresponding one-loop deformation of the global transformations is calculated in explicit form. The results obtained are exclusively general and take place for any bosonic or fermionic gauge theory with an irreducible closed gauge algebra and with an arbitrary (even open) non-anomalous algebra of global symmetry.

The paper is organized as follows. Section 2 is devoted to description of the general gauge theory with closed irreducible gauge algebra and fixing the notations and conventions. In Sect. 3 we describe the properties of the general gauge theory with global symmetry. In Sect. 4 we construct the quantum action of the theory under consideration and prove that the quantization leads to deformation of the classical global symmetries. In Sect. 5 we consider the above deformation in one-loop approximation and construct the deformation in an explicit form. Section 6 summarizes the results.

In the paper the DeWitt's condensed notations are used [35]. We employ the notation $\varepsilon(A)$ for the Grassmann parity of any quantity $A$. All derivatives with respect to sources are taken from the left only. The right and left derivatives with respect to fields are marked by special symbols " $\leftarrow$ " and " $\rightarrow$ " respectively. The symbol $A_{, i}(\varphi)$ means the right derivative of $A(\varphi)$ with respect to the field $\varphi^{i}$.

\section{General gauge theory: notations and conventions}

Consider the general gauge theory with closed irreducible gauge algebra. It means that the initial action, $S_{0}=S_{0}(\varphi)$, of the fields $\varphi=\left\{\varphi^{i}\right\}, \quad i=1,2, \ldots, n, \varepsilon\left(\varphi^{i}\right)=\varepsilon_{i}$ is invariant under the gauge transformations

$S_{0, i}(\varphi) R_{\alpha}^{i}(\varphi)=0, \quad \delta \varphi^{i}=R_{\alpha}^{i}(\varphi) \xi^{\alpha}$,

where $\xi^{\alpha}$ are the arbitrary functions of space-time coordinates ${ }^{1}$ with Grassmann parities $\varepsilon\left(\xi^{\alpha}\right) \equiv \varepsilon_{\alpha}$, and $R_{\alpha}^{i}=$ $R_{\alpha}^{i}(\varphi), \varepsilon\left(R_{\alpha}^{i}\right)=\varepsilon_{i}+\varepsilon_{\alpha}$ are generators of gauge transformations which are assumed to be linear independent in gauge indices $\alpha$. It is convenient to introduce the operators of gauge transformations, $\hat{R}_{\alpha}=\hat{R}_{\alpha}(\varphi)$,

$\hat{R}_{\alpha}=\frac{\overleftarrow{\delta}}{\delta \varphi^{i}} R_{\alpha}^{i}$

so that the gauge invariance of $S_{0}(2.1)$ is written in the form

$S_{0} \hat{R}_{\alpha}=0$.

The algebra of the gauge generators $\hat{R}_{\alpha}$ has the following form due to the closure:

$\left[\hat{R}_{\alpha}, \hat{R}_{\beta}\right]=-\hat{R}_{\gamma} F_{\alpha \beta}^{\gamma}$

or

$R_{\alpha, j}^{i} R_{\beta}^{j}-(-1)^{\varepsilon_{\alpha} \varepsilon_{\beta}} R_{\beta, j}^{i} R_{\alpha}^{j}=-R_{\gamma}^{i} F_{\alpha \beta}^{\gamma}$,

where $F^{\gamma}{ }_{\alpha \beta}=F^{\gamma}{ }_{\alpha \beta}(\varphi)$ are the structure coefficients depending in general on fields $\varphi$ and obeying the symmetry properties

$F^{\gamma}{ }_{\alpha \beta}=-(-1)^{\varepsilon_{\alpha} \varepsilon_{\beta}} F_{\beta \alpha}^{\gamma}$.

For Yang-Mills theories they are constants. The Jacobi identities written in terms of the gauge generators and the structure coefficients read

$\left(F_{\alpha \rho}^{\sigma} F_{\beta \gamma}^{\rho}+F_{\alpha \beta, i}^{\sigma} R_{\gamma}^{i}\right)(-1)^{\varepsilon_{\alpha} \varepsilon_{\gamma}}+\operatorname{cycle}(\alpha \beta \gamma)=0$.

For irreducible gauge theories the extended configuration space is described by the fields :

$\phi^{A}=\left(\varphi^{i}, B^{\alpha}, C^{\alpha}, \bar{C}^{\alpha}\right)$,

$\varepsilon\left(\varphi^{i}\right)=\varepsilon_{i}, \quad \varepsilon\left(B^{\alpha}\right)=\varepsilon_{\alpha}, \quad \varepsilon\left(C^{\alpha}\right)=\varepsilon\left(\bar{C}^{\alpha}\right)=\varepsilon_{\alpha}+1$,

$\left.\operatorname{gh}\left(\varphi^{i}\right)=\operatorname{gh}\left(B^{\alpha}\right)=0, \quad \operatorname{gh}\left(C^{\alpha}\right)=1, \quad \operatorname{gh} \bar{C}^{\alpha}\right)=-1$,

where $B^{\alpha}$ are Nakanishi-Lautrup auxiliary fields, $C^{\alpha}$ and $\bar{C}^{\alpha}$ are the ghost and anti-ghost fields. For gauge theories under consideration which belong to the rank 1 gauge theories in terminology of BV formalism [11,12] the total (quantum)

\footnotetext{
1 In DeWitt's condensed notation it means the index $\alpha$ includes spacetime coordinates among others.
} 
action, $S=S(\phi)$, can be written in the form of the FaddeevPopov action [36],

$S(\phi)=S_{0}(\varphi)+\bar{C}^{\alpha} \chi_{\alpha, i}(\varphi) R_{\beta}^{i}(\varphi) C^{\beta}+\chi_{\alpha}(\varphi) B^{\alpha}$

where $\chi_{\alpha}=\chi_{\alpha}(\varphi), \varepsilon\left(\chi_{\alpha}\right)=\varepsilon_{\alpha}$, are some gauge functions lifting the degeneracy of the classical gauge invariant action $S_{0}$.

The action (2.9) is invariant under the following BRST transformation

$\delta_{\mathrm{B}} S(\phi)=0, \quad \delta_{\mathrm{B}} \phi^{A}=R^{A}(\phi) \lambda$

with

$$
\begin{aligned}
R^{A}(\phi)= & \left(R_{\alpha}^{i}(\varphi) C^{\alpha}, 0,\right. \\
& \left.-\frac{1}{2}(-1)^{\varepsilon_{\beta}} F^{\alpha}{ }_{\beta \gamma}(\varphi) C^{\gamma} C^{\beta}, B^{\alpha}\right),
\end{aligned}
$$

where $\lambda$ is a constant Grassmann parameter $(\varepsilon(\lambda)=1)$. Introducing the operator of BRST transformations, $\hat{R}=\hat{R}(\phi)$, and using abbreviation $R^{A}=R^{A}(\phi)$

$\hat{R}=\frac{\overleftarrow{\delta}}{\delta \phi^{A}} R^{A}, \quad \varepsilon(\hat{R})=1$

the BRST invariance of action $S$ (2.10) can be written as

$S \hat{R}=0$.

With the help of $\hat{R}$ the action (2.9) rewrites in the form

$S=S_{0}+\Psi \hat{R}$,

where we have introduced the gauge fixing functional $\Psi=$ $\Psi(\phi)$ of the form

$\Psi(\phi)=\bar{C}^{\alpha} \chi_{\alpha}(\varphi), \quad \varepsilon(\Psi)=1$.

The quantum action in form of (2.14) is evidently BRST invariant due to the nilpotency of $\hat{R}, \hat{R}^{2}=0$.

\section{General gauge theory with rigid symmetry}

Let the initial action $S_{0}(\varphi)$ is invariant under the global symmetry transformations as well,

$S_{0, i}(\varphi) T_{a}^{i}(\varphi)=0, \quad \delta_{T} \varphi^{i}=T_{a}^{i}(\varphi) \omega^{a}$

where $\omega^{a}, a=1.2, \ldots, m$ are constant (not depending on space-time coordinates) parameters with Grassmann parities $\varepsilon\left(\omega^{a}\right) \equiv \varepsilon_{a}$, and $T_{a}^{i}=T_{a}^{i}(\varphi)\left(\varepsilon\left(T_{a}^{i}\right)=\varepsilon_{i}+\varepsilon_{a}\right)$ are generators of global transformations. We want to emphasize once more that the gauge transformations are characterized by the space-time dependent parameters while the parameters of the global transformations are space-time independent. In the framework of the condensed notations the gauge and global transformations look similar. To make the difference of two these types of the transformations explicit we denote the index of gauge parameter by greek letter and the index of global parameter by latin letter. We should keep in mind that summation in latin letters does not include a space-time integral. Let $\hat{T}=\hat{T}(\varphi)$,

$\hat{T}=\hat{T}_{a} \omega^{a}, \quad \hat{T}_{a}=\hat{T}_{a}(\varphi)=\frac{\overleftarrow{\delta}}{\delta \varphi^{i}} T_{a}^{i}$

be the operator of global transformations. Then, in general (see [22])

$\left[\hat{T}_{a}, \hat{T}_{b}\right]=-\hat{T}_{c} f_{a b}^{c}-\hat{R}_{\alpha} K_{a b}^{\alpha}-\frac{\overleftarrow{\delta}}{\delta \varphi^{i}} \lambda^{i j}{ }_{a b} S_{0, j}$,

or

$$
\begin{aligned}
& T_{a, j}^{i} T_{b}^{j}-(-1)^{\varepsilon_{a} \varepsilon_{b}} T_{b, j}^{i} T_{a}^{j} \\
& \quad=-T_{c}^{i} f_{a b}^{c}-R_{\alpha}^{i} K_{a b}^{\alpha}-\lambda_{a b}^{i j} S_{0, j},
\end{aligned}
$$

Here,

$f_{a b}^{c}=f_{a b}^{c}(\varphi), \quad f_{a b}^{c}=-f_{b a}^{c}(-1)^{\varepsilon_{a} \varepsilon_{b}}$,

$\varepsilon\left(f_{a b}^{c}\right)=\varepsilon_{a}+\varepsilon_{b}+\varepsilon_{c}$,

$K_{a b}^{\alpha}=K_{a b}^{\alpha}(\varphi), \quad K_{a b}^{\alpha}=-K_{b a}^{\alpha}(-1)^{\varepsilon_{a} \varepsilon_{b}}$,

$\varepsilon\left(K_{a b}^{\alpha}\right)=\varepsilon_{a}+\varepsilon_{b}+\varepsilon_{\alpha}$,

$\lambda^{i j}{ }_{a b}=\lambda^{i j}{ }_{a b}(\varphi), \quad \lambda^{i j}{ }_{a b}=-\lambda^{i j}{ }_{b a}(-1)^{\varepsilon_{a} \varepsilon_{b}}$,

$\varepsilon\left(\lambda^{i j}{ }_{a b}\right)=\varepsilon_{a}+\varepsilon_{b}+\varepsilon_{i}+\varepsilon_{j}$.

To close the algebra of gauge and global symmetries (2.5) and (3.4) we add the following relations

$\left[\hat{R}_{\alpha}, \hat{T}_{a}\right]=-\hat{R}_{\beta} U_{\alpha a}^{\beta}$,

or

$R_{\alpha, j}^{i} T_{b}^{j}-(-1)^{\varepsilon_{\alpha} \varepsilon_{b}} T_{b, j}^{i} R_{\alpha}^{j}=-R_{\beta}^{i} U_{\alpha b}^{\beta}$,

which means that the commutator of gauge and global transformations is some gauge transformation. The relations (2.4), (3.3), (3.8) express the algebraic structure of the set of local and global transformations in the general gauge theory. In particular, they show that the gauge transformations form the ideal in the commutator algebra of global and gauge transformations under consideration. The structure coefficients $U_{\alpha b}^{\beta}=U_{\alpha b}^{\beta}(\varphi)$ obey the symmetry properties

$U_{\alpha b}^{\beta}=-U_{b \alpha}^{\beta}(-1)^{\varepsilon_{\alpha} \varepsilon_{b}}$.

The Jacobi identities for global generators have the form

$$
\begin{aligned}
T_{d}^{i} & \left(f_{a e}^{d} f_{b c}^{e}+f_{a b, i}^{d} T_{c}^{i}\right)(-1)^{\varepsilon_{a} \varepsilon_{c}} \\
& +R_{\alpha}^{i}\left(U_{a \beta}^{\alpha} K_{b c}^{\beta}+K_{a b, i}^{\alpha} T_{c}^{i}\right)(-1)^{\varepsilon_{a} \varepsilon_{c}} \\
& +\left(T_{a, j}^{i} \lambda^{j k}{ }_{b c} S_{0, k}-\lambda^{i j}{ }_{a b} S_{0, j k} T_{c}^{k}\right. \\
& \left.-\lambda^{i j}{ }_{a b, k} S_{0, j} T_{c}^{k}(-1)^{\varepsilon_{j} \varepsilon_{k}}\right)(-1)^{\varepsilon_{a} \varepsilon_{c}} \\
& +\operatorname{cycle}(a, b, c)=0 .
\end{aligned}
$$


In the case of closed global transformations $\left(\lambda^{i j}{ }_{a b}=0\right)$ the Jacobi identity (3.11) splits into two relations

$$
\begin{aligned}
& \left(f_{a e}^{d} f_{b c}^{e}+f_{a b, i}^{d} T_{c}^{i}\right)(-1)^{\varepsilon_{a} \varepsilon_{c}}+\operatorname{cycle}(a, b, c)=0, \\
& \left(U_{a \beta}^{\alpha} K_{b c}^{\beta}+K_{a b, i}^{\alpha} T_{c}^{i}\right)(-1)^{\varepsilon_{a} \varepsilon_{c}}+\text { cycle }(a, b, c)=0 .
\end{aligned}
$$

Using the Jacobi identity for two global and one gauge transformations we obtain the following relations

$$
\begin{aligned}
& T_{c}^{i} f_{a b, i}^{c} R_{\alpha}^{i}(-1)^{\varepsilon_{\alpha} \varepsilon_{a}}+R_{\gamma}^{i}\left(\left(U^{\gamma}{ }_{\alpha c} f_{a b}^{c}\right.\right. \\
& \left.\quad+F^{\gamma}{ }_{\alpha \beta} K^{\gamma}{ }_{a b}\right)(-1)^{\varepsilon_{\alpha} \varepsilon_{b}}+K^{\gamma}{ }_{a b, j} R_{\alpha}^{j}(-1)^{\varepsilon_{\alpha} \varepsilon_{a}} \\
& \quad+\left(U^{\gamma}{ }_{\alpha a, j} T_{b}^{j}-U^{\gamma}{ }_{\alpha b, j} T_{a}^{j}(-1)^{\varepsilon_{a} \varepsilon_{\beta}}\right)(-1)^{\varepsilon_{\alpha} \varepsilon_{b}} \\
& \left.\quad-\left(U^{\gamma}{ }_{a \beta} U^{\beta}{ }_{b \alpha}-U^{\gamma}{ }_{b \beta} U^{\beta}{ }_{a \alpha}(-1)^{\varepsilon_{a} \varepsilon_{b}}\right)(-1)^{\varepsilon_{\alpha} \varepsilon_{a}}\right) \\
& \quad+\left(\lambda^{i j}{ }_{a b, k} R_{\alpha}^{k}(-1)^{\varepsilon_{\alpha}\left(\varepsilon_{a}+\varepsilon_{j}\right)}-R_{\alpha, k}^{i} \lambda^{k j}{ }_{a b}(-1)^{\varepsilon_{\alpha} \varepsilon_{b}}\right) S_{0, j} \\
& \quad+\lambda^{i j}{ }_{a b} S_{0, j k} R_{\alpha}^{k}(-1)^{\varepsilon_{\alpha} \varepsilon_{a}}=0 .
\end{aligned}
$$

For closed global transformations $\left(\lambda^{i j}{ }_{a b}=0\right)$ it follows from (3.14) that the structure coefficients $f_{a b}^{c}$ are gauge invariant,

$f_{a b, i}^{c} R_{\alpha}^{i}=0$,

and the following relations take place

$$
\begin{aligned}
& -\left(U_{a \beta}^{\gamma} U_{b \alpha}^{\beta}-U_{b \beta}^{\gamma} U_{a \alpha}^{\beta}(-1)^{\varepsilon_{a} \varepsilon_{b}}\right)(-1)^{\varepsilon_{\alpha}\left(\varepsilon_{a}+\varepsilon_{b}\right)} \\
& \quad+K_{a b, j}^{\gamma} R_{\alpha}^{j}(-1)^{\varepsilon_{\alpha}\left(\varepsilon_{a}+\varepsilon_{b}\right)} \\
& \quad+\left(U_{\alpha a, j}^{\gamma} T_{b}^{j}-U_{\alpha b, j}^{\gamma} T_{a}^{j}(-1)^{\varepsilon_{a} \varepsilon_{b}}\right) \\
& \quad+U_{\alpha c}^{\gamma} f_{a b}^{c}+F_{\alpha \beta}^{\gamma_{\alpha \beta} K_{a b}^{\gamma}=0 .}
\end{aligned}
$$

The Jacobi identity for operators $\hat{R}_{\alpha}, \hat{R}_{\beta}, \hat{T}_{a}$ lead to the relations

$$
\begin{aligned}
& F_{\alpha \beta, j}^{\sigma} T_{a}^{j}-U_{a \gamma}^{\sigma} F_{\alpha \beta}^{\gamma}(-1)^{\varepsilon_{a}\left(\varepsilon_{\alpha}+\varepsilon_{\beta}\right)} \\
& \quad+F_{\alpha \gamma}^{\sigma} U_{\beta a}^{\gamma}-F_{\beta \gamma}^{\sigma} U_{\alpha a}^{\gamma}(-1)^{\varepsilon_{\alpha} \varepsilon_{\beta}} \\
& \quad+U_{\beta a, j}^{\sigma} R_{\alpha}^{j}(-1)^{\varepsilon_{\alpha}\left(\varepsilon_{\beta}+\varepsilon_{a}\right)}-U_{\alpha a, j}^{\sigma} R_{\beta}^{j}(-1)^{\varepsilon_{a} \varepsilon_{\beta}}=0 .
\end{aligned}
$$

The relations (2.1), (2.4)-(2.7), (3.1)-(3.11), (3.14) and (3.17) describe structure and properties of symmetry algebra of the gauge system under consideration.

The operators $\hat{R}$ and $\hat{T}_{a}$ do not commute,

$$
\begin{aligned}
{\left[\hat{R}, \hat{T}_{a}\right]=} & -\hat{R}_{\beta} U_{\alpha a}^{\beta} C^{\alpha}(-1)^{\varepsilon_{a}} \\
& +\frac{\overleftarrow{\delta}}{\delta C^{\alpha}} \frac{1}{2}(-1)^{\varepsilon_{\beta}} F^{\alpha}{ }_{\beta \gamma, j} T_{a}^{j} C^{\gamma} C^{\beta}(-1)^{\varepsilon_{a}\left(\varepsilon_{\beta}+\varepsilon_{\gamma}\right)}
\end{aligned}
$$

From (3.18) we obtain the important relations

$\Psi\left[\hat{R}, \hat{T}_{a}\right]=-\Psi \hat{R}_{\beta} U_{\alpha a}^{\beta} C^{\alpha}(-1)^{\varepsilon_{a}}$.
The right-hand side in (3.19) is nothing but the gauge transformations of $\Psi$ with gauge parameters $\Lambda^{\beta}(\phi)=$ $U^{\beta}{ }_{\alpha a}(\varphi) C^{\alpha}(-1)^{\varepsilon_{a}} \omega^{a}$.

The quantum action (2.9) is not invariant under the global transformations (3.1). Using (3.19), the variation of $S=$ $S(\phi)$ can be presented in the form

$\delta_{T} S=S \hat{T}_{a} \omega^{a}=\left(\Psi \hat{T}_{a} \omega^{a}\right) \hat{R}+\left(\Psi \hat{R}_{\beta}\right) U_{\alpha a}^{\beta} \omega^{a} C^{\alpha}$.

The first term in the right-hand side of (3.20) describes the variation of gauge fixing functional $\Psi$ under global transformation while the second summand is the gauge transformation of $\Psi=\Psi(\phi)$ with local parameters $\Lambda^{\alpha}=\Lambda^{\alpha}(\phi)$,

$\Lambda^{\alpha}(\phi)=U_{\beta a}^{\alpha}(\varphi) \omega^{a} C^{\beta}$.

Second term in (3.20) can be presented in the form

$\left(\Psi \hat{R}_{\beta}\right) U_{\alpha a}^{\beta} \omega^{a} C^{\alpha}=S_{, \alpha} \Lambda^{\alpha}, \quad S_{, \alpha}=S \frac{\overleftarrow{\delta}}{\delta C^{\alpha}}$

It will allow us in the next section to analyze the (in)dependence of the effective action on the global symmetry transformations in the theory under consideration.

\section{Structure of global symmetries on quantum level}

In this section we consider properties of global symmetry within the BRST quantization taking into account that the status of the gauge symmetry is well-known. In particular, the vacuum functional, $\mathrm{Z}$,

$Z=Z_{\chi}=\int D \phi \exp \left\{\frac{i}{\hbar} S(\phi)\right\}$

does not depend on the choice of admissible gauge fixing functions $\chi_{\alpha}$ thanks to the BRST symmetry of $S(\phi)(2.10)$,

$\delta_{\chi} Z=0$.

In deriving this result the following conditions

$(-1)^{\varepsilon_{\beta}} F^{\beta}{ }_{\beta \alpha}(\varphi)=0, \quad(-1)^{\varepsilon_{i}} \frac{\vec{\delta}}{\delta \varphi^{i}} R_{\alpha}^{i}(\varphi)=0$

are used.

Let $Z_{T}$ be the vacuum functional for the theory with action $S(\phi)+\delta_{T} S(\phi)$

$Z_{T}=\int D \phi \exp \left\{\frac{i}{\hbar}\left[S(\phi)+\delta_{T} S(\phi)\right]\right\}$.

Making use in the functional integral (4.4) the change of variables in the form of BRST transformations (2.10) but with replacement $\lambda \rightarrow \Lambda(\phi)$ where

$\Lambda(\phi)=\frac{i}{\hbar} \bar{C}^{\alpha} \chi_{\alpha, i}(\varphi) T_{a}^{i}(\varphi) \omega^{a}=\frac{i}{\hbar} \Psi(\phi) \hat{T}(\varphi)$, 
and taking into account the triviality of Jacobian of such change, we arrive at the relation

$Z_{T}=\int D \phi \exp \left\{\frac{i}{\hbar}\left[S(\phi)+S_{, \alpha}(\phi) \Lambda^{\alpha}(\phi)\right]\right\}$.

Then performing the change of variables $C^{\alpha}$ in the form

$C^{\alpha} \rightarrow C^{\alpha}-U_{\beta a}^{\alpha}(\varphi) \omega^{a} C^{\beta}$

with the Jacobian equal to the unit and assuming the fulfilment of the conditions

$(-1)^{\varepsilon_{\alpha}} U_{\alpha a}^{\alpha}(\varphi)=0$,

we have the statement

$Z_{T}=Z$

i. e. the vacuum functional is invariant under the classical global transformations.

The generating functional of the Green functions, $Z(J)$, and the connected Green functions, $W(J)$, is represented by the functional integral

$$
\begin{aligned}
Z(J) & =\int D \phi \exp \left\{\frac{i}{\hbar}\left[S(\phi)+J_{A} \phi^{A}\right]\right\} \\
& =\exp \left\{\frac{i}{\hbar} W(J)\right\} .
\end{aligned}
$$

As a main consequence of the BRST symmetry of $S(\phi)$, there exits the Ward identity for $Z(J)$ and $W(J)$ in the form

$$
\begin{aligned}
& J_{A} R^{A}\left(\frac{\hbar}{i} \frac{\delta}{\delta J}\right) Z(J)=0, \\
& J_{A} R^{A}\left(\frac{\delta W}{\delta J}+\frac{\hbar}{i} \frac{\delta}{\delta J}\right) \cdot 1=0 .
\end{aligned}
$$

The generating functional of vertex functions (effective action), $\Gamma=\Gamma(\Phi)$, is defined standardly through the Legendre transformation of $W(J)$,

$\Gamma(\Phi)=W(J)-J_{A} \Phi^{A}, \quad \Phi^{A}=\frac{\delta W(J)}{\delta J_{A}}$,

so that

$\Gamma(\Phi) \frac{\overleftarrow{\delta}}{\delta \Phi^{A}}=-J_{A}$

The Ward identity (4.11) rewrites for $\Gamma(\Phi)$ in the form

$\Gamma(\Phi) \frac{\overleftarrow{\delta}}{\delta \Phi^{A}} \bar{R}^{A}(\Phi)=0$

where

$\bar{R}^{A}(\Phi)=R^{A}(\hat{\Phi}) \cdot 1$,

and

$\hat{\Phi}^{A}=\Phi^{A}+i \hbar\left(\Gamma^{\prime \prime}-1\right)^{A B}(\Phi) \frac{\vec{\delta}}{\delta \Phi^{B}}$.
In (4.16) the matrix $\left(\Gamma^{\prime \prime}-1\right)^{A B}(\Phi)$ is inverse to

$$
\begin{aligned}
& \left(\Gamma^{\prime \prime}\right)_{A B}(\Phi)=\frac{\vec{\delta}}{\delta \Phi^{A}}\left(\Gamma(\Phi) \frac{\overleftarrow{\delta}}{\delta \Phi^{B}}\right) \\
& \left(\Gamma^{\prime \prime}-1\right)^{A C}\left(\Gamma^{\prime \prime}\right)_{C B}=\delta_{B}^{A} .
\end{aligned}
$$

The Ward identity (4.14) can be interpreted as the invariance of effective action $\Gamma(\Phi)$ under the quantum BRST transformations of $\Phi^{A}$ with generators $\bar{R}^{A}(\Phi)$.

In the functional integral (4.10) we make the change of integration variables $\varphi^{i}$ in the form of global transformations (3.1). Then, using the conditions

$(-1)^{\varepsilon_{i}} \frac{\vec{\delta}}{\delta \varphi^{i}} T_{a}^{i}(\varphi)=0$,

we obtain

$Z(J)=\int D \phi \exp \left\{\frac{i}{\hbar}\left[S(\phi)+\delta_{T} S(\phi)+J_{A} \phi^{A}+j_{i} T_{a}^{i}(\varphi) \omega^{a}\right]\right\}$,

where $\delta_{T} S(\phi)$ is defined in (3.20) and $j_{i}$ are external sources to $\varphi^{i}$. The conditions (4.18) lead to that the Jacobian of change of variables in the functional integral (4.19) is equal to unit. By performing the change of variables $\phi^{A}$ in the form

$\phi^{A} \rightarrow \phi^{A}+R^{A}(\phi) \Lambda(\phi)$

with $\Lambda(\phi)$ given in (4.5), and then additionally the transformations (4.7), we find in the first order in $\omega^{a}$

$$
\begin{aligned}
\int & D \phi\left(j_{i} T_{a}^{i}(\varphi)+J_{A} R^{A}(\phi) \Lambda_{a}(\phi)\right. \\
& \left.+J_{\alpha_{(C)}} U_{a \beta}^{\alpha}(\varphi) C^{\beta}(-1)^{\varepsilon_{a}}\right) \exp \left\{\frac{i}{\hbar}\left[S(\phi)+J_{A} \phi^{A}\right]\right\}=0,
\end{aligned}
$$

where we took into account that the Jacobian of change of variables is trivial and $J_{\alpha_{(C)}}$ are sources to fields $C^{\alpha}$. The equation (4.21) rewrites

$$
\begin{aligned}
& {\left[j_{i} T_{a}^{i}\left(\frac{\hbar}{i} \frac{\delta}{\delta j}\right)+J_{A} R^{A}\left(\frac{\hbar}{i} \frac{\delta}{\delta J}\right) \Lambda_{a}\left(\frac{\hbar}{i} \frac{\delta}{\delta J}\right)\right.} \\
& \left.\quad+\frac{\hbar}{i} J_{\alpha_{(C)}} U_{a \beta}^{\alpha}\left(\frac{\hbar}{i} \frac{\delta}{\delta j}\right) \frac{\delta}{\delta J_{\beta_{(C)}}}(-1)^{\varepsilon_{a}}\right] Z(J)=0, \quad a=1,2, \ldots, m .
\end{aligned}
$$

In terms of the functional $W=W(J)$ the relations (4.22) read

$$
\begin{aligned}
j_{i} T_{a}^{i}\left(\frac{\delta W}{\delta j}+\frac{\hbar}{i} \frac{\delta}{\delta j}\right)+J_{A} R^{A}\left(\frac{\delta W}{\delta J}+\frac{\hbar}{i} \frac{\delta}{\delta J}\right) \Lambda_{a}\left(\frac{\delta W}{\delta J}+\frac{\hbar}{i} \frac{\delta}{\delta J}\right) \\
+J_{\alpha_{(C)}} U_{a \beta}^{\alpha}\left(\frac{\delta W}{\delta j}+\frac{\hbar}{i} \frac{\delta}{\delta j}\right) \frac{\delta W}{\delta J_{\beta_{(C)}}}(-1)^{\varepsilon_{a}}=0, \quad a=1,2, \ldots, m .
\end{aligned}
$$


Then in terms of $\Gamma(\Phi)$ the relations (4.23) can be presented in the form

$\Gamma(\Phi)\left(\frac{\overleftarrow{\delta}}{\delta \Phi^{i}} T_{a}^{i}(\Phi)+\frac{\overleftarrow{\delta}}{\delta \Phi^{A}} M_{a}^{A}(\Phi)+\frac{\overleftarrow{\delta}}{\delta \Phi^{\alpha_{(C)}}} U_{a}^{\alpha}(\Phi)\right)=0$

where the notations

$$
\begin{aligned}
& T_{a}^{i}(\Phi)=\left.T_{a}^{i}(\varphi)\right|_{\varphi^{i} \rightarrow \hat{\Phi}^{i}} 1, \quad M_{a}^{A}(\Phi)=R^{A}(\hat{\Phi}) \Lambda_{a}(\hat{\Phi}) \cdot 1, \\
& U_{a}^{\alpha}(\Phi)=\left.U_{a \beta}^{\alpha}(\varphi)\right|_{\varphi^{i} \rightarrow \hat{\Phi}^{i}} \Phi^{\beta_{(C)}}(-1)^{\varepsilon_{a}}, \\
& J_{A}=\left(j_{i}, J_{\alpha_{(B)}}, J_{\alpha_{(C)}}, J_{\alpha_{(\bar{C})}}\right) \text {, } \\
& \Phi^{A}=\left(\Phi^{i}, \Phi^{\alpha_{(B)}}, \Phi^{\alpha_{(C)}}, \Phi^{\alpha_{(\bar{C})}}\right),
\end{aligned}
$$

were used.

The relations (4.24) mean that the effective action is invariant under the quantum global transformation,

$\Gamma(\Phi) \frac{\overleftarrow{\delta}}{\delta \Phi^{A}} \bar{T}_{a}^{A}(\Phi)=0$

with the deformed generators

$$
\begin{aligned}
\bar{T}_{a}^{A}(\Phi)= & T_{a}^{i}(\Phi)+M_{a}^{i}(\Phi), 0, \\
& \left.M_{a}^{\alpha_{(C)}}(\Phi)+U_{a}^{\alpha}(\Phi), M_{a}^{\alpha_{(\bar{C})}}(\Phi)\right) .
\end{aligned}
$$

The proof of invariance (4.26) is based on using the change of variables (4.5) which are not analytical in loop expansion parameter $\hbar$. Therefore, unlike the study of gauge dependence of effective action in the framework of BRST-BV formalism, the derivation of (4.27) is not related to the change of gauge functions. In the next section we will however prove that the correctness of (4.26) in loop expansion procedure is correct.

\section{Deformation of the global transformations in one-loop approximation}

As we pointed out at the end of Sect. 4, the change of variables (4.5) is non-analytical in $\hbar$ and the use of loop expansion looks doubtful. However, we will show that the one-loop approximation still works.

Consider the relations (4.26) in loop approximation. For the effective action we have

$\Gamma(\Phi)=S(\Phi)+\hbar \Gamma_{1}(\Phi)+O\left(\hbar^{2}\right)$.

The generators $\bar{T}_{a}^{A}(\Phi)$ are written in the same approximation as follows

$$
\begin{aligned}
\bar{T}_{a}^{A}(\Phi)= & \frac{1}{\hbar} R^{A}(\Phi) \tilde{\Lambda}(\Phi)+\bar{T}_{(0) a}^{A}(\Phi) \\
& +\hbar \bar{T}_{(1) a}^{A}(\Phi)+O\left(\hbar^{2}\right), \quad \tilde{\Lambda}(\Phi)=\hbar \Lambda(\Phi) .
\end{aligned}
$$

Due to the invariance of quantum action $S(\Phi)$ (2.9) under the BRST transformations (2.10), the first item in the right-hand side of (5.2), which is non-analytical in $\hbar$, does not contribute in the relations (4.26). As a result in zero loop approximations these relation take the form

$\frac{\delta S(\Phi)}{\delta \Phi^{A}} \bar{T}_{(0) a}^{A}(\Phi)+\frac{\delta \Gamma_{1}(\Phi)}{\delta \Phi^{A}} R^{A}(\Phi) \tilde{\Lambda}(\Phi)=0$.

In the next order we get

$S(\Phi) \frac{\overleftarrow{\delta}}{\delta \Phi^{A}} \bar{T}_{(1) a}^{A}(\Phi)+\Gamma_{1}(\Phi) \frac{\overleftarrow{\delta}}{\delta \Phi^{A}} \bar{T}_{(0) a}^{A}(\Phi)=0$

Now let us take into account the Ward identity for $\Gamma(\Phi)$ (4.14), then in the first order in $\hbar$ we have

$\Gamma_{1}(\Phi) \frac{\overleftarrow{\delta}}{\delta \Phi^{A}} R^{A}(\Phi)=0$

With this result the relations (5.3) rewrites

$S(\Phi) \frac{\overleftarrow{\delta}}{\delta \Phi^{A}} \bar{T}_{(0) a}^{A}(\Phi)=0$

These relations demonstrate that the quantum action $S(\Phi)$ is one-loop invariant under the deformed global symmetry transformations. In particular, the generators of this global symmetry in the sector of fields $\Phi^{i}$ have the form

$$
\begin{aligned}
\bar{T}_{(0) a}^{i}(\Phi)= & \left.\bar{T}_{(0) a}^{i}(\phi)\right|_{\phi \rightarrow \Phi}, \quad \bar{T}_{(0) a}^{i}(\phi)=T_{a}^{i}(\varphi) \\
& -R_{\alpha}^{i}(\varphi) \Sigma_{(0) a}^{\alpha}(\phi)-R_{\alpha, j}^{i}(\varphi) \Theta_{(0) a}^{j \alpha}(\phi),
\end{aligned}
$$

where the following notations

$$
\begin{aligned}
& \Sigma_{(0) a}^{\alpha}(\phi)=\left(C^{\alpha}\left(S^{\prime \prime}-1\right)^{\left.\beta_{(\bar{C}}\right)^{j}}(\phi)\right. \\
& \left.+\left(S^{\prime \prime}-1\right)^{\alpha_{(C)} j}(\phi) \bar{C}^{\beta}(-1)^{\varepsilon_{j}\left(\varepsilon_{\beta}+1\right)}\right) N_{\beta a, j}(\varphi) \\
& +\left(S^{\prime \prime}-1\right)^{\alpha_{(C)} \beta_{(\bar{C})}}(\phi) \chi_{\beta, k j}(\varphi) T_{a}^{j}(\varphi) \\
& +C^{\alpha} \bar{C}^{\beta} \chi_{\beta, j k}(\varphi)\left(S^{\prime \prime}-1\right)^{k l}(\phi) T_{a, l}^{j}(\varphi)(-1)^{\varepsilon_{l}\left(\varepsilon_{j}+\varepsilon_{a}+1\right)}, \\
& \Theta_{(0) a}^{j \alpha}(\phi)=\left(\left(S^{\prime \prime-1}\right)^{j \alpha_{(C)}}(\phi) \bar{C}^{\beta}\right.
\end{aligned}
$$

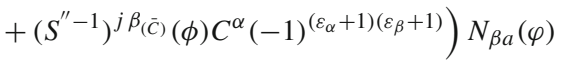

$$
\begin{aligned}
& +\left(S^{\prime \prime}-1\right)^{j l}(\phi) C^{\alpha} \bar{C}^{\beta} N_{\beta a, l}(\varphi)(-1)^{\varepsilon_{l}\left(\varepsilon_{\alpha}+\varepsilon_{\beta}\right)}, \\
& N_{\beta a}(\varphi)=\chi_{\beta, k}(\varphi) T_{a}^{k}(\varphi) \text {. }
\end{aligned}
$$

are introduced. The deformation of global generators $T_{a}^{i}(\varphi)$, defined in initial configuration space $\{\varphi\}$, looks very nontrivial already in the one-loop approximation. Such a deformation is done in the full configuration space of the gauge theory (2.8) with the help of generators $R_{\alpha}^{i}(\varphi)$ of gauge symmetry of initial action (2.1) and its derivatives $R_{\alpha, j}^{i}(\varphi)$. The relations (2.1), (3.1) and (5.7) lead to

$S_{0, i}(\varphi) \bar{T}_{(0) a}^{i}(\phi)=-S_{0, i}(\varphi) R_{\alpha, j}^{i}(\varphi) \Theta_{(0) a}^{j \alpha}(\phi) \neq 0$.

It means non invariance of an initial action $S_{0}(\varphi)$ under the deformed global transformations. 


\section{Summary}

In the present paper we have studied the general problem of global (rigid) symmetries in quantum general gauge theory with closed irreducible gauge algebra. Using the BRSTBV quantization procedure $[11,12]$ we have constructed a deformation of global symmetry generators so that the full quantum action of the initial gauge theory and hence the effective action are invariant under such deformed global transformations. This statement is valid for any bosonic or fermionic gauge theory with an irreducible and closed algebra of gauge transformations and with an arbitrary (even open) non-anomalous algebra of global symmetry. Form of the deformed global symmetry generators in one-loop approximation is calculated in the explicit form. Note that from algebraic point of view the deformation of global symmetry generators, studied in the present paper, is similar to the known phenomena of the deformation of gauge generators under renormalization procedure (see e.g. $[13,37,38]$ ), although the mechanisms of deformation differ.

In general the BRST-BV technique involves introduction for every field of the full configuration space the corresponding antifield with opposite Grassmann parity. It allows to study the many properties of the gauge theory on quantum level in general terms. However, to simplify the consideration in this paper we derived all the results in the configuration space of fields only (2.8). Generalization of the results obtained for extended configuration space of fields and antifields can be done in the same method.

Problem of global symmetries in quantum general gauge theories is discussed in earlier papers [19-22] however the accents were aimed on the other aspects. First, in [19] it was shown the possibility to construct the Lagrangian models possessing the gauge and supersymmetry invariance under the assumption that the global supersymmetry transformations obey the closed algebra. In our paper we work with an arbitrary (including open) global (super)symmetry of a given gauge theory. Also we suppose the absence of anomalies in combined gauge and global algebra of generators but in principle it is possible to study the problem of coexistence of gauge and global symmetries in presence of anomalies using the approach of work [20]. Of course the implementation of this program requires a separate independent study. Second, in paper [21] it was shown that the attempts to construct an action invariant under BRST- and closed rigid symmetries lead to the breakdown of non-degeneracy of the full quantum action. In our paper we proved that this problem is resolved on the base of quantum deformation of global generators. Third, in paper [22] it was shown that the global symmetries of an initial gauge action in the field-antifield formalism can be extended to include the all fields and the all antifields. After that the authors of [22] introduced a constant ghost for each global symmetry and modified the master-equation to incorporate the global symmetries into its solutions but they did not discuss the deformations of the global symmetry generators. It is clear that this approach differs from our consideration since from the beginning we work only in the configuration field space and derive the above deformations.

The general enough approach to the problem of global symmetries in the quantum gauge theory was proposed in the work [32] under assumptions that the initial classical theory consists of only bosonic fields and the algebra of the classical global transformations is closed. In our paper we have considered more general case of the theory with bosonic and fermionic fields and open algebra of the global (super)symmetries. However, what is very important, we work with more general effective action than in [32]. In our case, the sources are introduced not only for initial fields but also for ghost and auxiliary fields. This allowed us to describe the deformation of the generators completely in the algebraic terms.

The global (super)symmetric and gauge transformations have been studied in the framework of singular gauge fixing procedure. In practice it is more convenient to use a nonsingular gauges. All our basic statements are still valid in this case as well. We should only modify the quantum action (2.9) in the form

$$
\begin{aligned}
S(\phi)= & S_{0}(\varphi)+\bar{C}^{\alpha} \chi_{\alpha, i}(\varphi) R_{\beta}^{i}(\varphi) C^{\beta} \\
& +\chi_{\alpha}(\varphi) B^{\alpha}+\frac{\xi}{2} B^{\alpha} B^{\alpha},
\end{aligned}
$$

where $\xi$ is a gauge parameter. Due to the property of BRST transformations (2.11) $B^{\alpha} \hat{R}=0$, the action (6.1) remains invariant under the BRST transformations, $S \hat{R}=0$. Thus, from a principled point of view the results obtained will be the same.

Acknowledgements The authors thank I.V. Tyutin for useful discussions and S.M. Kuzenko for correspondence. The research was supported in parts by Russian Ministry of Education and Science, Project No. 3.1386.2017. The authors are also grateful to RFBR Grant, Project No. 18-02-00153 for partial support.

Open Access This article is distributed under the terms of the Creative Commons Attribution 4.0 International License (http://creativecomm ons.org/licenses/by/4.0/), which permits unrestricted use, distribution, and reproduction in any medium, provided you give appropriate credit to the original author(s) and the source, provide a link to the Creative Commons license, and indicate if changes were made. Funded by SCOAP . $^{3}$

\section{References}

1. C. Becchi, A. Rouet, R. Stora, Renormalization of the abelian Higgs-Kibble model. Commun. Math. Phys. 42, 127 (1975)

2. I.V. Tyutin, Gauge Invariance in Field Theory and Statistical Physics in Operator Formalism, vol. 39 (Lebedev Inst., Moscow, 1975) (preprint) 
3. D.Z. Freedman, P. van Nieuwenhuizen, S. Ferrara, Progress toward a theory of supergravity. Phys. Rev. D 13, 3214 (1976)

4. S. Deser, B. Zumino, Consitent supergravity. Phys. Lett. B 62, 335 (1976)

5. N.K. Nielsen, Ghost counting in supergravity. Nucl. Phys. B 140, 494 (1978)

6. R.E. Kallosh, Modified rules in supergravity. Nucl. Phys. B 141, 141 (1978)

7. P.K. Townsend, Covariant quantization of antisymmetric gauge fields. Phys. Lett. B 88, 97 (1979)

8. B. de Wit, J.W. van Holten, Covariant quantization of gauge theories with open algebra. Phys. Lett. B 79, 389 (1978)

9. E.S. Fradkin, G.A. Vilkovisky, Quantization of relativistic systems with constraints. Phys. Lett. B55, 224 (1975)

10. I.A. Batalin, G.A. Vilkovisky, Relativistic S matrix of dynamical systems with Boson and Fermion constraints. Phys. Lett. B69, 309 (1977)

11. I.A. Batalin, G.A. Vilkovisky, Gauge algebra and quantization. Phys. Lett. B 102, 27 (1981)

12. I.A. Batalin, G.A. Vilkovisky, Quantization of gauge theories with linearly dependent generators. Phys. Rev. D 28, 2567 (1983)

13. B.L. Voronov, P.M. Lavrov, I.V. Tyutin, Canonical transformations and gauge dependence in general gauge theories. Sov. J. Nucl. Phys. 36, 292 (1982)

14. J. Gomis, S. Weinberg, Are nonrenormalizable gauge theories renormalizable. Nucl. Phys. B 469, 473 (1996)

15. G. Barnich, On the quantum Batalin-Vilkovisky formalism and renormalization of nonlinear symmetries. JHEP 9903, 010 (1999)

16. M.E. Peskin, D.V. Schroeder, An Introduction to Quantum Field Theory (Perseus Books, New York, 1995)

17. M.B. Green, J.H. Schwarz, E. Witten, Superstring Theory (Cambridge University Press, Cambridge, 1987)

18. E. D'Hoker, D.Z. Freedman, Supersymmetric Gauge Theories and AdS/CFT Correspondence, TASI 2001 Lecture Notes (2004). arXiv: hep-th/02012523

19. B. de Wit, D.Z. Freedman, Combined supersymmetric and gaugeinvariant field theories. Phys. Rev. D 12, 2286 (1975)

20. L. Bonora, P. Pasti, M. Tonin, ABJ anomalies in supersymmetric Yang-Mills theories. Phys. Lett. B 156, 341 (1985)

21. J.W. van Holten, Rigid symmetries and BRST-invariance in gauge theories. Phys. Lett. B 200, 507 (1988)

22. F. Brandt, M. Henneaux, A. Wilch, Global symmetries in the antifield formalism. Phys. Lett. B 387, 320 (1996)

23. I.L. Buchbinder, S.M. Kuzenko, Ideas and Methods of Supersymmetry and Supergravity (IOP Publishing, Bristol, 1998)
24. A.S. Galperin, E.A. Ivanov, V.I. Ogievetsky, E.S. Sokatchev, Harmonic Superspace (Cambridge University Press, Cambridge, 2001)

25. G. Bossard, E. Ivanov, A. Smilga, Ultraviolet behavior of 6D supersymmetric Yang-Mills theories and harmonic superspace. JHEP 1512, 085 (2015). arXiv:1509.08027 [hep-th]

26. I.L. Buchbinder, E.I. Buchbinder, S.M. Kuzenko, B.A. Ovrut, Background field method for $\mathrm{N}=2$ super Yang-Mills theories in Harmonic Superspace. Phys. Lett. B 417, 61 (1998). arXiv:hep-th/9704214

27. E.I. Buchbinder, I.L. Buchbinder, S.M. Kuzenko, Nonholomorphic effective potential in $\mathrm{N}=4$, SU(n) SYM. Phys. Lett. B 446, 216 (1998). arXiv:hep-th/9810239

28. I.L. Buchbinder, E.A. Ivanov, N.G. Pletnev, Superfield approach to the construction of effective action in quantum field theory with extended supersymmetry. Phys. Part. Nucl. 47, 291 (2016)

29. I.L. Buchbinder, E.A. Ivanov, I.B. Samsonov, The low-energy $\mathcal{N}=4$ SYM effective action in diverse harmonic superspaces. Phys. Part. Nucl. 48, 333 (2017). arXiv:1603.02768 [hep-th]

30. I.L. Buchbinder, E.A. Ivanov, B.S. Merzlikin, K.V. Stepanyantz, One-loop divergences in $6 D, \mathcal{N}=(1,0)$ SYM theory. JHEP 01, 128 (2017). arXiv: 1612.03190 [hep-th]

31. I.L. Buchbinder, E.A. Ivanov, B.S. Merzlikin, K.V. Stepanyantz, Supergraph analysis of the one-loop divergences in $6 D, \mathcal{N}=(1,0)$ and $\mathcal{N}=(1,1)$ gauge theories. Nucl. Phys. B 921, 127 (2017). arXiv: 1704.02530 [hep-th]

32. S.M. Kuzenko, I.N. McArthur, Quantum metamorphosis of conformal symmetry in $\mathrm{N}=4$ super Yang-Mills theory. Nucl. Phys. B 640, 78 (2002). arXiv:hep-th/0203236

33. S.M. Kuzenko, I.N. McArthur, On quantum deformation of conformal symmetry: Gauge dependence via field redefinitions. Phys. Lett. B 544, 357 (2002). arXiv:hep-th/0206234

34. S.M. Kuzenko, I.N. McArthur, S. Theisen, Low-energy dynamics from deformed conformal symmetry in quantum 4-D N $=2$ SCFTS. Nucl. Phys. B 660, 131 (2003). arXiv:hep-th/0210007

35. B.S. DeWitt, Dynamical Theory of Groups and Fields (Gordon and Breach, Philadelphia, 1965)

36. L.D. Faddeev, V.N. Popov, Feynman diagrams for the Yang-Mills field. Phys. Lett. B 25, 29 (1967)

37. P.M. Lavrov, I.V. Tyutin, On the structure of renormalization in gauge theories. Sov. J. Nucl. Phys. 34, 156 (1981)

38. P.M. Lavrov, I.V. Tyutin, On the generating functional for the vertex functions in Yang-Mills theories. Sov. J. Nucl. Phys. 34, 474 (1981) 